\title{
Active longitudes in sunspot activity: Century scale persistence
}

\author{
S. V. Berdyugina ${ }^{1,2}$ and I. G. Usoskin ${ }^{3}$ \\ 1 Institut für Astronomie, ETHZ, 8092 Zürich, Switzerland \\ 2 Astronomy Division, PO Box 3000, 90014 University of Oulu, Finland \\ e-mail: sveta.berdyugina@oulu.fi \\ 3 Sodankylä Geophysical Observatory (Oulu unit), 90014 University of Oulu, Finland \\ e-mail: ilya.usoskin@oulu.fi
}

Received 6 November 2002 / Accepted 12 May 2003

\begin{abstract}
A novel analysis of sunspot group data for the past 120 years reveals that sunspots in both northern and southern hemispheres are formed preferably in two persistent active longitudes separated by $180^{\circ}$. In the Carrington reference frame, the active longitudes continuously migrate in phase with respect to the Carrington meridian with a variable rate. They remain however a quasi-rigid structure. We find that the migration of the active longitudes is determined by changes of the mean latitude of sunspots and the differential rotation. The differential rotation rate calculated from the migration is in agreement with SOHO/MDI measurements. The two active longitude periodically alternate being the dominant region, similar to the "flip-flop" phenomenon known in starspot activity. The period of the oscillations is 3.8 and 3.65 years in the north and south, respectively. The difference between the periods is significant and can be related to the known north-south asymmetry in the solar magnetic activity. Our results provide new observational constraints for current solar dynamo models and strengthen the solar paradigm for magnetic activity on cool stars.
\end{abstract}

Key words. Sun: activity - Sun: magnetic fields - sunspots - stars: activity

\section{Introduction}

The distribution of sunspots in the solar photosphere reflects the distribution of magnetic fields in the convection zone and provides strong observational constraints on the solar dynamo theory (e.g., Brandenburg 2001 and references therein). Sunspots are known to appear preferably in narrow latitudinal belts and approach the equator as the solar 11-year cycle advances, the pattern known as the Maunder butterfly diagram.

A longitudinal behaviour of sunspot activity does not show such a clear pattern. Many attempts were undertaken to find preferred longitudes of sunspot formation, so-called active longitudes, using various weighting and smoothing methods (e.g., Chidambara 1932; Losh 1939; Lopez Arroyo 1961; Warwick 1965; Vitinskij 1969; Balthasar \& Schüssler 1983) as well as the autocorrelation function of sunspot numbers (Bogart 1982). Although all the above authors reported evidences for the existence of longitudinal inhomogeneities in sunspot distribution during some time intervals, the results were inconsistent as concerned the number of the active longitudes, their lifetime, location and rotation rate. Typically, the reported active longitudes were evident for several years but disappeared on longer time-scales or appeared at different location. Signatures of possible longitudinal inhomogeneities were also reported in

Send offprint requests to: S. V. Berdyugina,

e-mail: sveta@astro.phys.ethz.ch distributions of solar flares, in the solar corona, solar wind and interplanetary magnetic field (e.g., Bumba \& Obridko 1969; Jetsu et al. 1997; Benevolenskaya et al. 1999; Neugebauer et al. 2000). Note that most of earlier researchers assumed that the active longitudes is a structure which is fixed in Carrington coordinates, i.e. rotating with a constant rate. Some authors, however, found that the rotation rate of active longitudes may vary in time (e.g., Lopez Arroyo 1961; Stanek 1972; Bogart 1982; Ozkan \& Derman 1982).

Recently, persistent active longitudes separated by about $180^{\circ}$ have been detected on different types of cool active stars, including a young solar analogue (e.g., Berdyugina \& Tuominen 1998; Rodonò et al. 2000; Berdyugina et al. 2002; Korhonen et al. 2002). In many cases, the active longitudes have been found to migrate in longitude as a rigid structure, seemingly due to the star's differential rotation. This suggests that solar active longitudes, if they exist, may also follow the surface differential rotation, and the assumption on their even rotation made by previous investigators is probably invalid.

In the present paper, we perform a novel analysis of sunspot data for the past 120 years and search for large-scale sunspot formation regions persistent on long time scales. Our main results have been first reported by Berdyugina \& Usoskin (2002) and are updated by the present paper. Here we analyze the data with two different filtering techniques which produce similar results. The data and the methods are presented in Sect. 2. 
Avoiding the misleading assumption on even rotation of the activity regions, we are able to recover a dynamic pattern of the active longitudes which changes its rotation rate in accord with sunspot mean latitude changes and the differential rotation. Our new findings on the active longitudes are presented in Sect. 3. Finally, in Sect. 4, we summarize our results, compare the active longitude patterns on the Sun and cool stars and discuss implications of our results for solar and stellar physics.

\section{Data and filtering techniques}

For our analysis we use daily data on sunspot group locations and areas collected at the Royal Greenwich Observatory, the US Air Force and the National Oceanic and Atmospheric Administration for the years 1874-2001, covering 11 full solar cycles. Because of the known asymmetry between the northern and southern hemispheres (e.g., Verma 1993), we analyze the data from the two hemispheres separately. In order to reveal large-scale long-living structures in sunspot longitudinal distribution, we use two different techniques: (1) averaging sunspot group area over fixed longitude bins and (2) calculating expected solar brightness variations due to spots. The first technique, thus, averages the sunspot data with a rectangular filter, while the second one smoothes them with a bell-shaped sliding window.

\subsection{The rectangular filter}

Averaging sunspot data with a rectangular filter is a simple and, therefore, most common technique used in previous studies. Here we carry out a similar analysis and average the sunspot area data with a fixed $20^{\circ}$ rectangular window in longitude. However, unlike the previous researchers, we average the data for each solar rotation separately, with one rotation defined as Carrington rotation $(\mathrm{CR}=27 \mathrm{~d} .2753)$. Averaging on such a short time-scale allows us to recover locations of sunspot clusters which are not smeared by the differential rotation and assumed to be constant during one CR.

For each CR we built a vector $S$ consisting of 18 elements, which are equal to the sum of sunspot areas in corresponding $20^{\circ}$ longitude bins over all latitudes. Then this vector is normalized to the total sunspot area observed during the $\mathrm{CR}$ :

$W_{i}=\frac{S_{i}}{\sum_{i} S_{i}}, \quad i=1, \ldots, 18$

Such normalization allows us to study active longitudes at different activity levels. For an even longitudinal distribution of sunspots, the value of $W$ should be 0.055 , and it is higher in those longitude bins where the sunspot activity is clustered. We assume that deviations from an even longitudinal distribution are significant if $W>0.11$. An example of such averaging is shown in Fig. 1b for the CR 1808. Three peaks in the histogram with $W>0.11$ correspond to large spot clusters in the original data shown in Fig. 1a. We read out longitudes of such peaks from the histograms for further analysis.
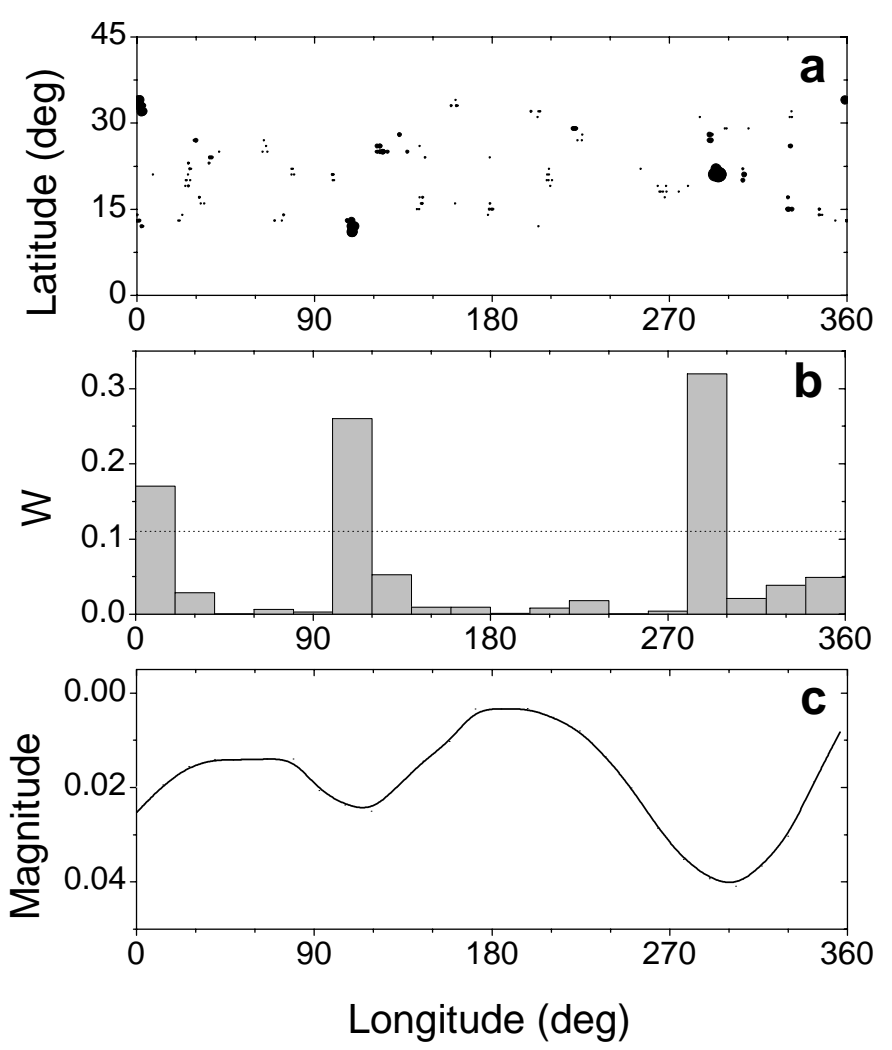

Fig. 1. An illustration of the active longitude analysis applied to sunspot areas in the northern solar hemisphere during the Carrington rotation 1808 (October to November 1988). a) Sunspot distribution on the solar surface (areas are enlarged by a factor of 50). b) Normalized sunspot areas $W$ in $20^{\circ}$ longitudinal bins (rectangular filter). The dotted line corresponds to the cut-off level of 0.11 . c) Synthesized solar brightness variations due to spots (bell-shaped filter).

\subsection{The bell-shaped filter}

The sunspot visibility on the solar surface is a bell-shaped function which has the maximum at the centre of the solar disk and decreases towards the limb. Therefore, expected daily variations of the solar brightness due to dark spots are equivalent to data smoothed with such a filter sliding in time. In order to perform such smoothing, for each CR we build a temperature distribution on the solar surface defined by cool dark spots with $T_{\mathrm{sp}}=4000 \mathrm{~K}$ on the bright surface with $T_{\mathrm{ph}}=5750 \mathrm{~K}$ (Fig. 1a). Then, we synthesize a light curve in relative bolometric magnitudes as follows:

$m=-2.5 \log \left[\frac{(1-f) T_{\mathrm{ph}}^{4}+f T_{\mathrm{sp}}^{4}}{T_{\mathrm{ph}}^{4}}\right]$,

where $f$ is a filling factor of spots visible on the solar disk at a given moment. It is calculated as a weighted sum of the spotted area over the visible solar surface:

$f=\frac{\sum \delta_{k} a_{k} d_{k}}{\sum a_{k} d_{k}}$

Here, $a_{k}$ is an elemental area at a given location, $d_{k}=1-$ $X+X \cos \theta_{k}$ is the limb darkening at the angular distance $\theta_{k}$ from the disk centre, and $\delta_{k}=1$ if there is a spot at a given location or $\delta_{k}=0$ otherwise. The summation is done with a 
grid $1^{\circ} \times 1^{\circ}$. The limb darkening coefficient $X=0.7$ is used. Note that the synthesized magnitudes differ from the real solar brightness since the latter includes also such bright phenomena as faculae.

The above procedure transforms the two-dimensional sunspot distribution into a one-dimensional data set and, therefore, is similar to other filtering techniques. An example of the synthesized light curve is presented in Fig. 1c. Minima in the light curve correspond to spot concentrations in Fig. 1a and peaks in Fig. 1b. We conclude therefore that the bell-shaped filter allows for effective and proper averaging of very detailed sunspot observations and reveals large-scale patterns of the sunspot activity. In contrast with the rectangular filter, it provides smooth longitudinal variations in the sunspot distribution. The longitudes of the light-curve minima are compared with those obtained with the rectangular filter (e.g., Fig. 1) and used in the further analysis.

Note that a similar analysis of light curves was successfully used for studying stellar active longitudes (e.g., Berdyugina \& Tuominen 1998; Rodonò et al. 2000; Berdyugina et al. 2002). Brightness minima in stellar light curves were associated with large spot concentrations and interpreted in terms of the spot filling factor, while the real spot distribution remained uncovered. The present calculations for sunspots suggest that the spot distribution on the stellar surfaces can be as complex as on the Sun. On the other hand, our new approach allows us to study the Sun as we would study a distant star whose large-scale magnetic activity only can be resolved. This makes it possible to compare the present results with those obtained for starspots.

\section{Results}

\subsection{Active longitudes}

Comparing the longitudes of sunspot clusters obtained with the two different filtering techniques, we find that the methods produce similar results (see Fig. 2). Therefore, we conclude that our results are method independent. For further analysis, we transform the Carrington longitudes, $\lambda$, into phases of the Carrington period: $\varphi=\lambda / 360^{\circ}$. Note that the phase derived in this way is $\leq 1$.

We plot the recovered phases of sunspot clusters versus time and find that the regions migrate in phase as rigid structures. When a region reaches $\varphi=1$, it appears again near $\varphi=0$. In such cases we add an integer to the phase and unfold continuous migration of the regions. An example for the solar cycle 18 is shown in Fig. 2. The migration results in a phase lag of about 2.5 rotations over a solar cycle. Although the recovered phases are somewhat scattered, they tend to form two parallel migration paths separated by 0.5 in phase. These paths can be called active longitudes since the regions migrate as a rigid structure, though with a variable rate.

In order to smooth out the scatter of individual points, we perform averaging of the recovered phases over half a year, separately for the two migration paths. The result is shown in Figs. 3 and 4 for all cycles in the northern and southern hemispheres, respectively. We find that (1) the active longitudes separated by 0.5 in phase are persistent in both hemispheres for

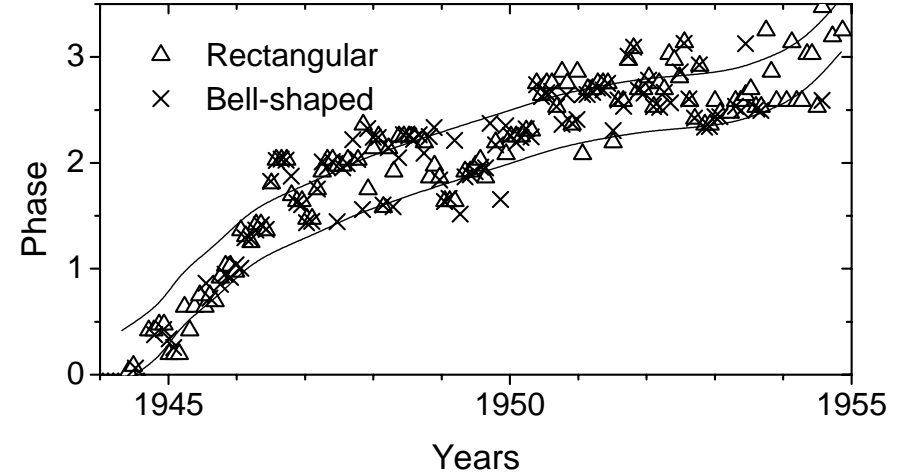

Fig. 2. Phases of the largest spot clusters obtained with the two different filtering techniques for the solar cycle 18 in the northern hemisphere. Lines correspond to the migration defined by the surface differential rotation and changes of the mean sunspot latitude. They indicate locations of the active longitudes separated by 0.5 in phase $\left(180^{\circ}\right.$ in longitude). Note that the major spot activity alternates the active longitudes in about $1.5-3$ years.

at least 120 years, (2) the migration continues throughout the entire studied period and results in a phase lag of about 28 rotations for 120 years, and (3) there is no breaks in the active longitudes during the solar minima.

In order to estimate the significance of the two active longitudes, we built histograms of the phases recovered for individual Carrington rotations (as shown in Fig. 2) with respect to semi-annual averages of one (lower) active longitude (shown in Figs. 3 and 4). The histograms are presented in Fig. 5. A clear two-peak distribution confirms the presence of the two active longitudes separated by 0.5 in phase, and its nearly double-Gaussian shape implies that individual spots are randomly spread around the preferred longitudes.

\subsection{Migration of the active longitudes}

\subsubsection{Long-term migration}

The recovered phases of the active longitudes are calculated for the Carrington rotation period, which corresponds to the mean synodic rotation period of sunspots and, according to the surface differential rotation, can be in principle attributed to the latitude of about $20^{\circ}$. Therefore, the observed lag of the active longitudes of 28 rotations for 120 years can be considered with respect to this latitude. The presence of such a lag indicates, however, that the long-term mean latitude of sunspot formation is somewhat lower and, thus, a shorter period would be more appropriate.

From our data we find that the mean latitude of sunspots over all studied cycles is about $14^{\circ}$ in both northern and southern hemispheres. This results in the period of about 26.9 days. With this period the linear part of the migration would be removed and the slope of the paths in Figs. 2-4 would change. However, the choice of the period does not effect the existence and separation of the active longitudes.

The relation of the active longitude migration to the mean latitude of sunspots is further confirmed by long-term variations of the migration rate in the north and south. We find that 

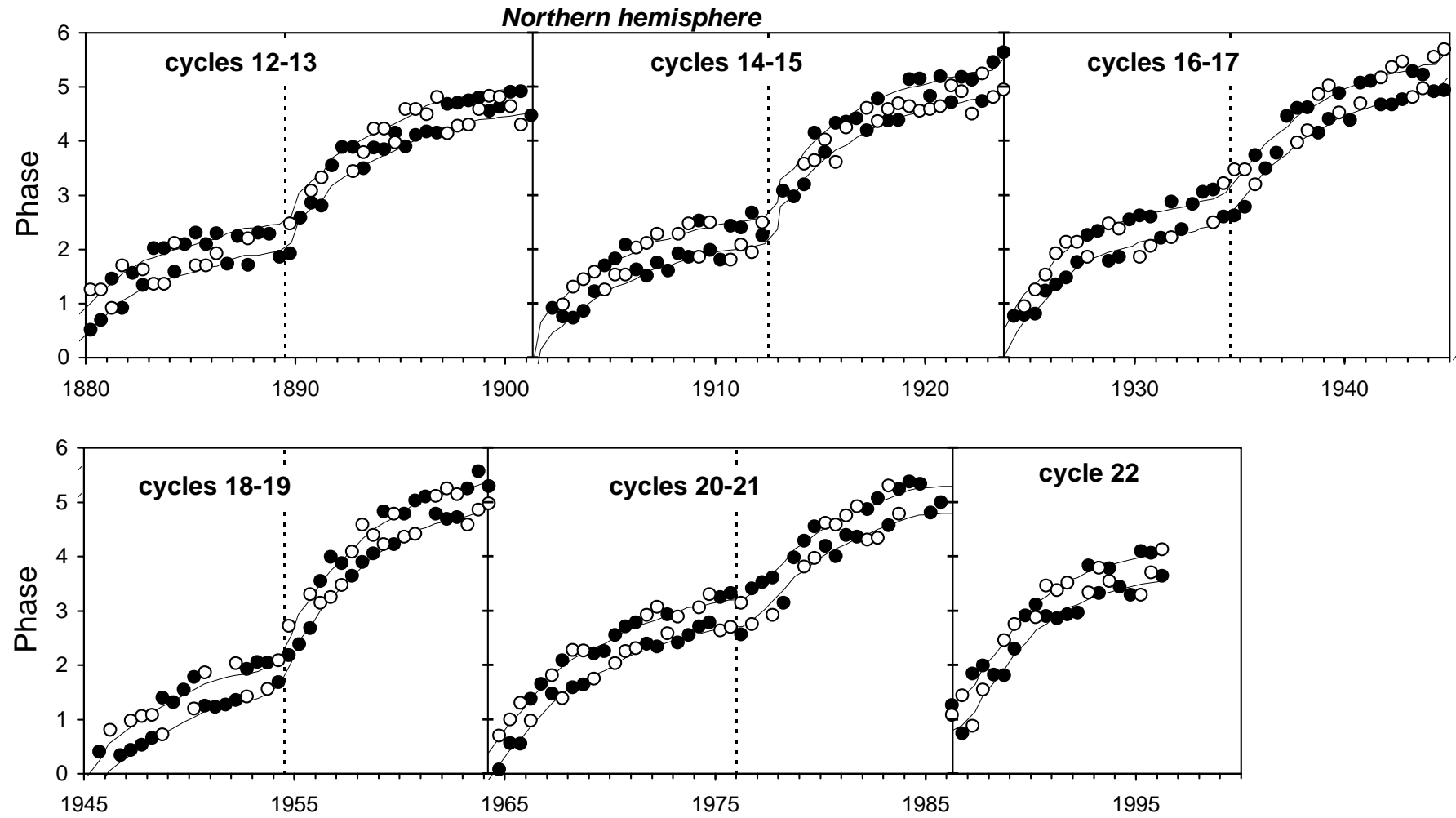

Fig. 3. Semi-annual phases of regions of preferred sunspot formation for solar cycles 12 to 22 in the northern hemisphere. Filled circles denote the phases of the active longitude, which is dominant during the corresponding 6 months, and open circles denote the other active longitude. Curves depict the phase migration resulting from a combination of changes of the mean latitude of sunspots and the differential rotation. Vertical lines separate the solar cycles. The migration continues over all 11 cycle and the active longitude delay for about 2.5 rotations per cycle. For better visibility the migration in the plots is discontinued every second cycle.

the mean latitude of sunspot formation is different for different cycles and varies by $\pm 1.5^{\circ}$ with a period of about 90 years. This is in agreement with findings by Pulkkinen et al. (1999), who also found that the difference between the mean latitudes in the north and south also varies with the 90 -year cycle. Such variations result in changes of the mean rotation period and, thus, variations of the mean migration rate from cycle to cycle. The difference in the migration rates between the north and south is removed on the time-scale of 90 years.

\subsubsection{Short-term migration}

As seen from Figs. 2-4, the migration rate of the active longitudes also varies on a time-scale of the solar cycle. In the beginning of the cycle the phase lag increases more rapid than in the end. Since in the beginning of the cycle sunspots are formed preferably at higher latitudes and approach the equator as the solar cycle advances, the change in the migration rate of the active longitudes can be related to the mean latitude of sunspots and the differential rotation.

The surface angular velocity at a latitude $\psi$ is described by the following approximation (Stix 1989):

$\Omega=A+B \sin ^{2} \psi$

where $A$ is the rotation rate at the equator and $B$ is the differential rotation rate. The latter can be determined from the observed phase lags of the active longitudes (Figs. 3 and 4). Then, an expected lag in the Carrington longitude, $\Delta \lambda$, is determined by changes of the mean latitude $\psi$ during the time interval $\Delta t$ as follows:

$\Delta \lambda(\Delta t)=B \int_{\Delta t} \sin ^{2} \psi(t) \mathrm{d} t$

Using the mean latitudes of sunspots obtained from the data, we calculate values of the above integral for each solar cycle. Comparing them with the obtained phase lags we find $B=-3.46 \pm 0.06 \mathrm{degr} /$ day (Fig. 6). This is in agreement with the value of $-3.43 \mathrm{degr} /$ day obtained from SOHO/MDI measurements (Schou et al. 1998). Such an agreement confirms that the migration of the active longitudes is indeed determined by changes of the sunspot mean latitudes and the differential rotation.

The effect of the differential rotation on the migration of the active longitudes can be understood if we neglect it and try to reproduce the earlier results (e.g., Losh 1939; Warwick 1965; Vitinskij 1969; Balthasar \& Schüssler 1983). For this purpose we assume that the active longitudes rotate with a constant rate corresponding to the Carrington period, i.e. $B=0$ and the phase lag for any time interval is zero (see Eq. (5)). Such an assumption is equivalent to averaging of sunspot longitudes on time-scales much longer than the Carrington rotation. For instance, a histogram of sunspot locations with the neglected migration for one solar cycle reveals only one significant active longitude (Fig. 7a). It becomes however less pronounced on the time-scale of two solar cycles (Fig. 7b) and completely 

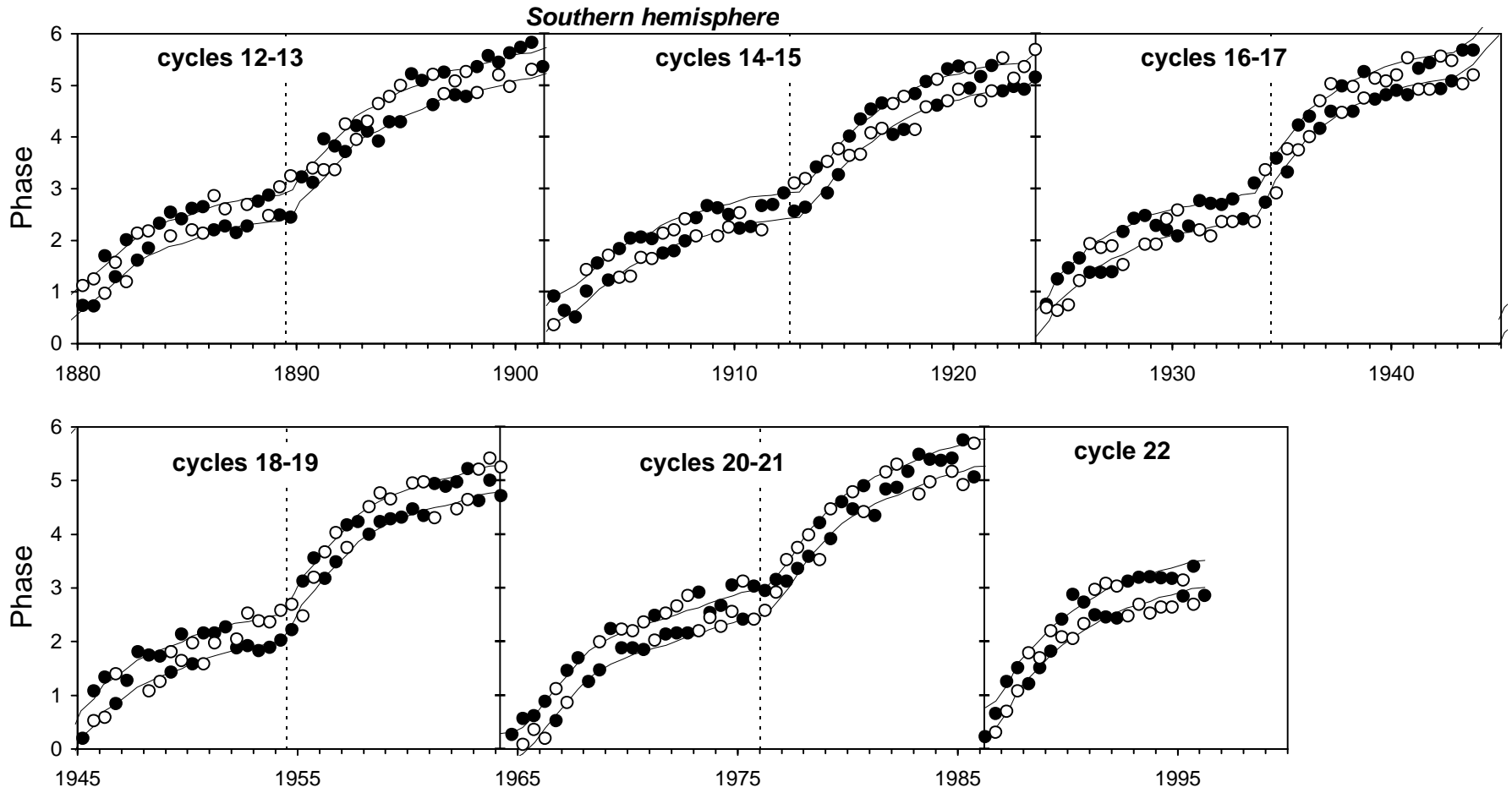

Fig. 4. The same as Fig. 3 for the southern hemisphere.

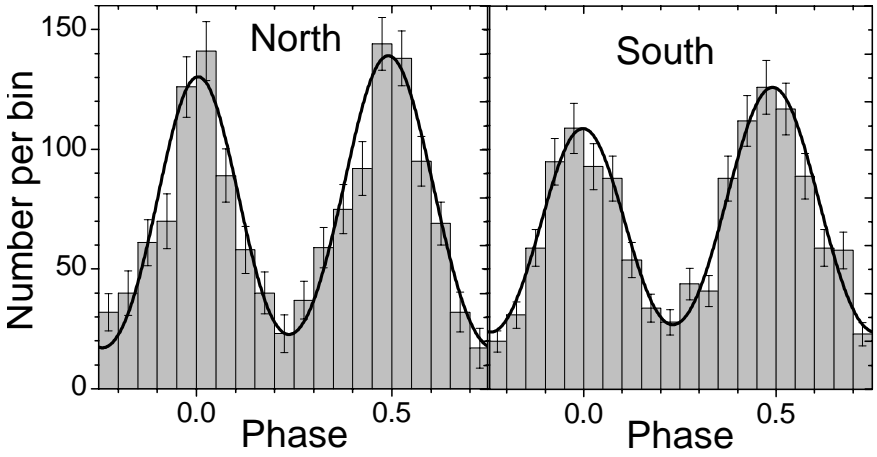

Fig. 5. Histogram distribution of recovered spot locations with respect to the semi-annual averages of one active longitude for solar cycles 12 to 22. Error bars correspond to $1 \sigma$ statistical error. The best-fit double Gaussian function with peaks at phases 0.0 and 0.5 and the standard deviation of about 0.11 is shown by solid lines.

washed out on longer time-scales. The probability that the spot distribution shown in Fig. 7b is defined by statistical fluctuations of the uniform distribution is as high as 0.75 . Therefore, we conclude that the assumption on the even rotation of the active longitudes as well as averaging of sunspot locations on long time-scales destroy the pattern of the active longitudes.

\subsection{Alternation of the active longitudes}

We notice that spots are preferably concentrated in one of the two active longitude, which is dominant at a given time. This is well seen, for instance, in Fig. 2, where only the largest spot concentrations are plotted for the solar cycle 18 in the northern hemisphere. In the beginning of the cycle, during about 1.5 years, the activity dominates the lower active

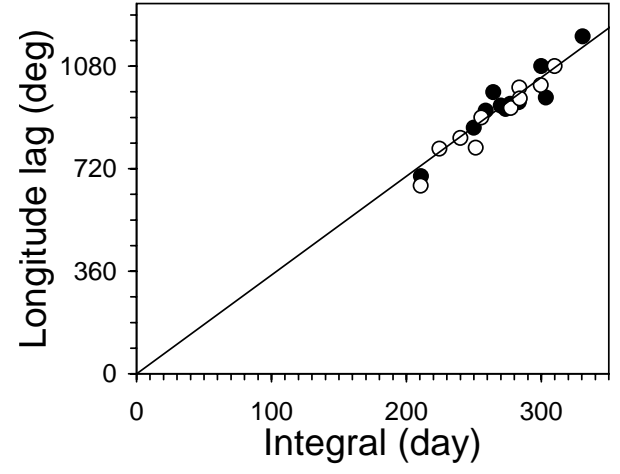

Fig. 6. Scatter plot of the longitude lag $\Delta \lambda(\Delta t)$ vs. the value of the integral $\int_{\Delta t} \sin ^{2} \psi(t) \mathrm{d} t$ (see Eq. (5)) for solar cycles 12 to 22 together with the best fit linear relation $B=-3.46 \mathrm{degr} /$ day (solid line). Solid and open dots correspond to the northern and southern hemispheres, respectively.

longitude. Then, during the cycle, it switches up and down several times and dominates the two active longitudes in turns for 1.5-3 years. Such alternations happen during other solar cycles as well, with about the same regularity in both hemispheres (Figs. 3 and 4).

In order to describe this phenomenon quantitatively, we build a series of the activity dominance $D$, so that for each Carrington rotation $D$ takes the value 0 or 1 , if the longitude at earlier or later phase dominates, respectively. In Figs. 2-4 the lower migration path corresponds to the active longitudes at earlier phase, and vice versa. By calculating a yearly running mean, we smooth the discrete $D$-series into a continuous series shown in Fig. 8a. The smoothed series reveals a clear periodic pattern for both hemispheres. The power spectrum of 

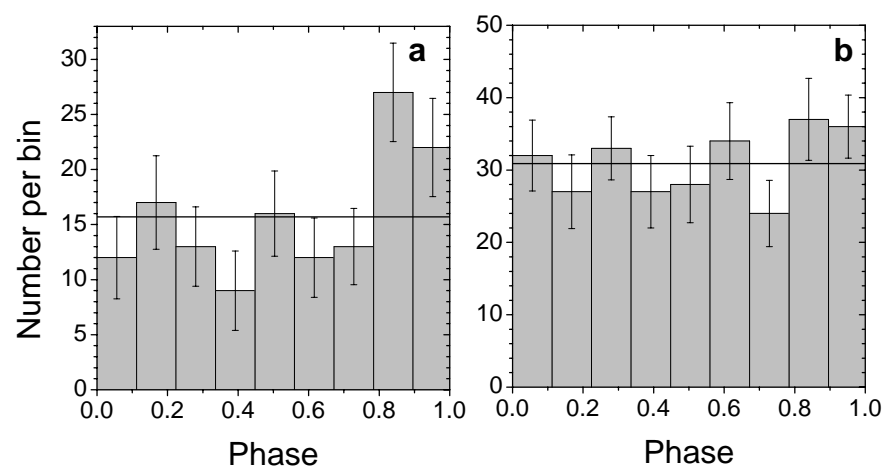

Fig. 7. Histogram distribution of recovered spot locations in the northern hemisphere assuming an even rotation with $B=0$ : a) for the solar cycle 22 and $\mathbf{b})$ for the cycles 21-22. Error bars correspond to $1 \sigma$ statistical error. The horizontal line corresponds to the uniform distribution.

the smoothed dominance shown in Fig. 8b exhibits main peaks at periods of 3.8 and 3.65 years in the northern and southern hemispheres, respectively. Both peaks are well above the $95 \%$ confidence level calculated against the spectrum of white noise with the same dispersion as the dominance series (e.g., Jenkins $\&$ Watts 1968). This implies that the two longitudes alternate periodically the dominant activity in about $1.8-1.9$ years on average. The individual periods of one longitude's dominance may last 1.5 to 3 years, while a switch of the activity from one longitude to the other is rather quick and may happen within a month (see, e.g., in Fig. 2).

On average 6 switches of the activity occurs during the 11-yr sunspot cycle and, thus, the 3.7-yr cycle is about $1 / 3$ of it. It is known however that the length of the sunspot cycle varies on a century time-scale. For instance, the recent solar cycles 18-22 were shorter than average and lasted about 10.4 years. We notice that the alternation cycle for these years is also shorter, about 3.3-3.5 years. This suggests, therefore, that the ratio $1 / 3$ is preserved on a long-time scale. The relation between the cycle lengths will be further investigated in more detail in a forthcoming paper.

The difference between the northern 3.8-yr and southern 3.65-yr cycles appears to be significant. If this difference is retained for all studied cycles, a beating period between the two frequencies should be observed: $1 / P_{\text {beat }}=1 / 3.65-1 / 3.8$, resulting in $P_{\text {beat }} \approx 90$ years. A running cross-correlation of the two smoothed dominances for the north and south indeed reveals a significant power near the period of 100 years, although the accuracy is limited by the length of the series. Power spectra of the cross-correlation functions for running windows of $130 \mathrm{CR}$ and $50 \mathrm{CR}$ are shown in Fig. 9. The power spectra reveals also that the period around 20 years appears to be significant. This suggests for the presence of the north-south asymmetry which is modulated on time-scales of 20 and 100 years, which is in agreement with the previous results (e.g., Verma 1993). We will study this modulation in more detail in a forthcoming paper.

\section{Discussion and conclusions}

Current models of the solar dynamo suggest that cyclonic turbulence in the outer solar convection zone generates a global poloidal magnetic field with its lines of force in meridional planes. Lines of the poloidal field are stretched by the differential rotation at the bottom of the convection zone where a strong toroidal magnetic field circling the solar axis is produced (e.g., Stix 1989). Because of buoyancy instabilities, portions of the toroidal field rise through the convection zone and form sunspots (Schüssler et al. 1994; Caligari et al. 1995). Therefore, sunspots reflect the evolution of the global magnetic field and can be used for studying the processes driving the magnetic field.

We analyzed the sunspot group data for nearly 120 years with two different filtering techniques, one averaging sunspot group area over fixed longitude bins with a rectangular filter, the other smoothing them with a sliding bell-shaped filter by calculating expected solar brightness variations due to spots. We found that both techniques produce similar results and concluded that the results are method independent. Our novel findings can be summarized as follows:

- Sunspots in both northern and southern hemispheres are preferably formed around two active longitudes separated by $180^{\circ}$. For the first time, we show that they are persistent for at least 120 years, which is the time span of the data.

- The two active longitudes is a long-lived quasi-rigid structure, although they are not fixed in any reference frame because of the differential rotation. They continuously migrate with respect to a chosen reference frame with a variable rate. In the Carrington reference frame, the migration results in a phase lag of about 2.5 solar rotations per sunspot cycle, in total about 28 rotations for 120 years.

- The migration of the active longitudes is caused by changes of the mean latitude of the sunspot formation and the differential rotation. Since sunspots are first formed at higher latitudes and approach the equator as the solar cycle advances, in the Carrington reference frame the migration is more rapid in the beginning of the cycle and slows down towards the end. The rate of the differential rotation $-3.46 \mathrm{deg} / \mathrm{day}$ obtained from the migration rate is in agreement with the recent measurements by SOHO/MDI.

- Neglecting the migration results in complete smearing of the active longitude pattern on time scales of more than one solar cycle. This explains the diversity and contradictions of the previosly published results.

- The major spot activity alternates the active longitudes in about 1.5-3 years. In the northern and southern hemispheres this results in the alternation cycles of 3.8 and 3.65 years, respectively, which is about $1 / 3$ of the sunspot 11 -yr cycle. The difference between the cycle lengths is significant and produces the beating effect between the north and south on the century time scale. The $1 / 3$ ratio seems to preserve on a long-time scale. 

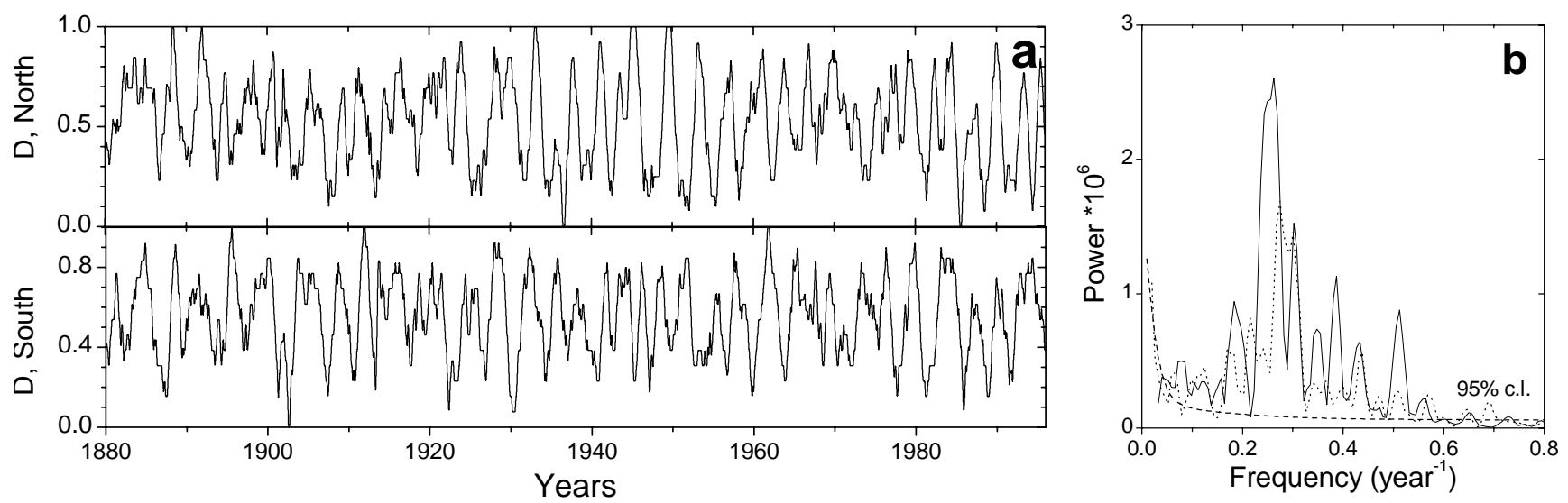

Fig. 8. Yearly smoothed dominance of the two active longitudes in the northern and southern hemispheres a) and corresponding power spectra b). The dashed line in the panel b) indicates the $95 \%$ confidence level.

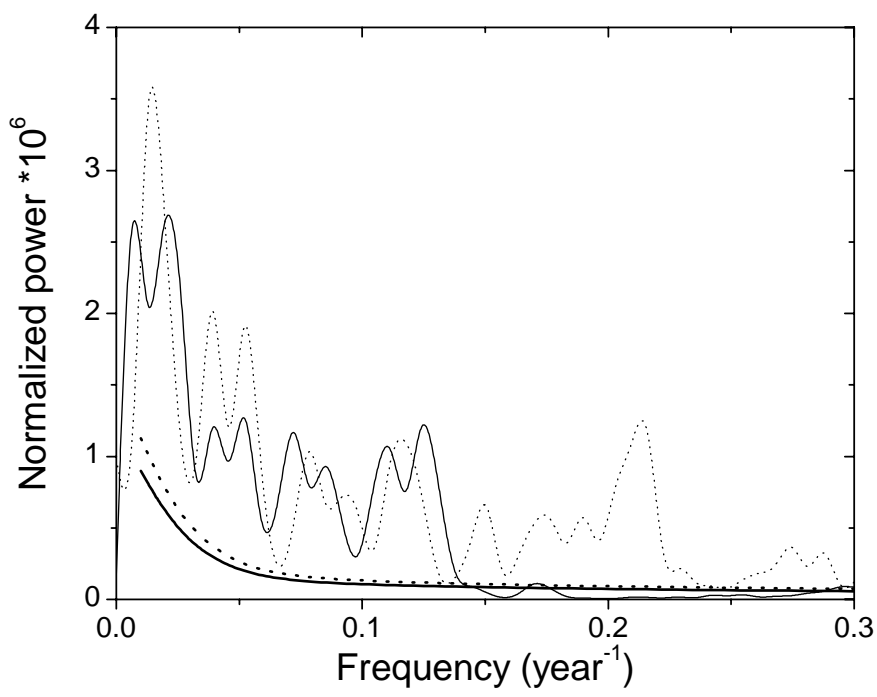

Fig. 9. Power spectra of the running cross-correlation function of the two smoothed dominance series for the northern and southern hemispheres shown in Fig. 8a. Spectra shown with thin solid and dotted lines correspond to the cross-correlation functions calculated with windows of $130 \mathrm{CR}$ and $50 \mathrm{CR}$, respectively. Smooth thick-line curves below the spectra indicate corresponding $95 \%$ confidence levels against the spectrum of white noise.

In terms of the mean-field dynamo theory, stable active longitudes separated by $180^{\circ}$ in the Sun and cool active stars can be explained as a result of excitation of a global nonaxisymmetric (quadrupole) dynamo mode (e.g., Moss et al. 1995; Tuominen et al. 2002). According to the latest solar dynamo model with a plausible solar rotation law (Moss \& Brooke 2000), a quadrupole mode should exist in the Sun along with the dipole. Moreover, the energy may oscillate between the dipole and quadrupole fields on various time scales. On the other hand, Schüssler et al. (1994) showed that buoyancy instability with the parity $m=2$ at the bottom of the convection zone is preferred, that can also result in active longitudes.

The fact that the active longitudes migrate with the surface differential rotation implies that flux ropes disconnect from their source regions at some time after the emergence. A period of the connection appears to be about life-time of individual spot groups, as they migrate with different rates (D'Silva \& Howard 1994) and can be seen moving across the active longitude migration. New groups appear, however, near the advanced active longitudes. This suggests, therefore, that it is the places of preferred flux emergence that migrate at the local photospheric rate. In fact, the same behaviour is observed in sunspot latitudes: it is the places of new sunspot emergence rather than spots themselves that migrate towards the equator during the solar cycle. Therefore, we conclude that the latitudinal and longitudinal migrations are driven by the same process in the Sun.

Periodic alternations of active longitudes are known in stellar activity as a "flip-flop" phenomenon. It was first detected on the single, rapidly rotating giant FK Com as a sudden shift of the activity by $180^{\circ}$ in longitude (Jetsu et al. 1991). The cyclic nature of the phenomenon was discovered by Berdyugina \& Tuominen (1998) who detected persistent active longitudes and a sequence of "flip-flops" on active giants in binary systems of RS CVn-type. Later, similar patterns were detected on very young (60 Myr) solar analogues LQ Hya (Berdyugina et al. 2002) and AB Dor (Järvinen et al. 2003) and also confirmed on FK Com (Korhonen et al. 2002). It was suggested that the switches of the dominant activity between the active longitudes are tracers of a new type of stellar magnetic cycles, which appear very different from, e.g., the sunspot cycle. Such cycles have not been yet encountered in stellar dynamo models. Our discovery of the "flip-flop" phenomenon on the Sun provides the opportunity to study it in detail.

The migration of the active longitudes on cool stars with respect to a chosen reference frame is almost linear for binary components and shows non-linear effects on single, rapidly rotating stars such as FK Com, AB Dor, and LQ Hya, which seemingly possess larger differential rotation rates. The solar differential rotation rate is however the largest among the others. Despite the diversity of the stellar ages, internal structure, rotational rates, etc., the similarity of the activity patterns on cool stars and the Sun suggest for a similar dynamo excited in these stars.

Signatures of the solar migrating active longitudes and of their 3.7-year oscillations can be searched also in chromospheric and coronal activity and in the heliosphere. 
For instance, the active longitude alternation may be responsible for the recently found phase variations of the interplanetary magnetic field with a period of about 3.2 years (Takalo $\&$ Mursula 2002). Also, rapid switches of the dominant activity between the active longitudes in 1.5-3 years may be related to quasi-biennial oscillations in the solar activity, which appear, e.g., in oscillations of the global solar magnetic field (Obridko \& Shelting 2001). Further, the difference in the alternation cycles in the north and south can be related to the observed north-south asymmetry of sunspot activity, such as systematic southward displacement of the magnetic equator (Bravo \& Stewart 1995; Mursula et al. 2001). The beating period of about 90 years between the two frequencies in the north and south is likely related to the Gleissberg cycle.

The fact that the 3.7-yr active longitude cycle is $1 / 3$ of the $11-y r$ sunspot cycle is probably of fundamental importance, as the same relation between analogous cycles has been found for a very active, young solar analogue LQ Hya (Berdyugina et al. 2002). A comparison of LQ Hya and other young solartype dwarfs with the present Sun allows for studying the evolution of the solar magnetic activity since the Sun arrived at the Main Sequence. The magnetic activity of LQ Hya was found to be driven by two dynamo modes, dipole and quadrupole, which maintain the cycles of 15 and 5.2 years, respectively (Berdyugina et al. 2002). Since LQ Hya can represent the Sun at the age of about $60 \mathrm{Myr}$, it was suggested that the activity of the young, rapidly rotating Sun could also consist of the two dynamo modes. During the solar evolution, as the Sun was losing its angular momentum, the power of the higher order mode should have significantly decreased. Our present results argue however that the quadrupole mode is still excited in the Sun. Also, it is very probable that the Sun possessed both 11-yr and 3.7-yr cycles since the beginning of its evolution on the Main Sequence.

Finally, the fact that the Sun shows the same pattern of magnetic activity as highly active stars strengthens the solar paradigm for magnetic activity on cool stars, allowing the physics of magnetic activity on such stars to be understood within the context of the physical principles deduced from the study of the Sun.

Acknowledgements. We thank G. Isaak, A. G. Kosovichev, M. Schüssler, S. K. Solanki and I. Tuominen for useful discussions of the present study. Solar data have been obtained from the GRO and USAF/NOAA web site

http://science.nasa.gov/ssl/pad/solar/greenwch.htm

\section{References}

Balthasar, H., \& Schüssler, M. 1983, Sol. Phys., 87, 23

Benevolenskaya, E. E., Kosovichev, A. G., \& Scherrer, P. H. 1999, Sol. Phys., 190, 145
Berdyugina, S. V., \& Tuominen, I. 1998, A\&A, 336, L25

Berdyugina, S. V., \& Usoskin, I. G. 2002, in 1st Potsdam Thinkshop, Sunspots and Starspots, Poster Proceedings, ed. K. G. Strassmeier, \& A. Washuettl (Golm: Brandenburgische Universitätsdruckerei), 31

Berdyugina, S. V., Pelt, J., \& Tuominen, I. 2002, A\&A, 394, 505

Bogart, R. S. 1982, Sol. Phys., 76, 155

Brandenburg, A. 2001, IAU Symp. 203, ed. P. Brekke, B. Fleck, \& J. B. Gurman, 144

Bravo, S., \& Stewart, G. 1995, ApJ, 446, 431

Bumba, V., \& Obridko, V. N. 1969, Sol. Phys., 6, 104

Caligari, P., Moreno-Insertis, F., \& Schüssler, M. 1995, ApJ, 441, 886

Chidambara, A. 1932, MNRAS, 93, 150

D’Silva, S., \& Howard, R. 1994, Sol. Phys., 151, 213

Järvinen, S. P., Berdyugina, S. V., \& Tuominen, I. 2003, A\&A, submitted

Jenkins, G. M., \& Watts, D. G. 1968, Spectral analysis and its applications (London: Holden-Day)

Jetsu, L., Pelt, J., Tuominen, I., \& Nations, H. 1991, in The Sun and Cool Stars: Activity, Magnetism, Dynamos, ed. I. Tuominen, D. Moss, \& G. Rüdiger, IAU Coll. 130, Lect. Not. Phys., vol. 380 (Berlin: Springer), 381

Jetsu, L., Pohjolainen, S., Pelt., J., \& Tuominen, I. 1997, A\&A, 318, 293

Korhonen, H., Berdyugina, S. V., \& Tuominen, I. 2002, A\&A, 390, 179

Lopez Arroyo, M. 1961, The Observatory, 81, 205

Losh, H. M. 1939, Pub. Obs. Univ. Michigan, 7, 127

Moss, D., Barker, D. M., Brandenburg, A., \& Tuominen, I. 1995, A\&A, 294, 155

Moss, D., \& Brooke, J. 2000, MNRAS, 315, 521

Mursula, K., Usoskin, I. G., \& Kovaltsov, G. A. 2001, Sol. Phys., 198, 51

Neugebauer, M., Smith, E. J., Ruzmaikin, A., Feynman, J., \& Vaughan, A. H. 2000, J. Geophys. Res., 105, 2315

Obridko, V. N., \& Shelting, B. D. 2001, Astron. Rep., 45, 1012

Ozkan, T., \& E. Derman, 1982, Astrophys. Space Sci., 87, 211

Pulkkinen, P. J., Brooke, J., Pelt, J., \& Tuominen, I. 1999, A\&A, 341, L43

Rodonò, M., Messina, S., Lanza, A. F., Cutispoto, G., \& Teriaca, L. 2000, A\&A, 358, 624

Schüssler, M., Caligari, P., Ferriz-Mas, A., \& Moreno-Insertis, F. 1994, A\&A, 281, L69

Schou, J., Antia, H. M., Basu, S., et al. 1998, ApJ, 505, 390

Stanek, W. 1972, Sol. Phys., 27, 89

Stix, M. 1989, The Sun: An Introduction (Berlin: Springer-Verlag)

Tuominen, I., Berdyugina, S. V., \& Korpi, M. J. 2002, Astron. Nachr., 323,367

Takalo, J., \& K. Mursula, K. 2002, Geophys. Res. Lett., 29, 31

Verma, V. K. 1993, ApJ, 403, 797

Vitinskij, Yu. I. 1969, Sol. Phys., 7, 210

Vitinsky, Yu. I., Kopecky, M., \& Kuklin, G. V. 1986, Statistics of Sunspot Activity (Moscow: Nauka)

Warwick, C. S. 1965, ApJ, 141, 500 